\title{
波前弥合现象对地震层析成像的影响
}

\author{
刘玉柱"，董良国 \\ 同济大学海洋地质国家重点实验室, 上海 200092 \\ *E-mail: liuyuzhu@tongji.edu.cn
}

收稿日期: 2011-03-29; 接受日期: 2012-02-04

国家自然科学基金(批准号：40804023)、国家高技术研究发展计划(编号：2008AA093001)和国家重点基础研究发展计划(编号： 2006CB202402)资助

\begin{abstract}
摘要 波前弥合是地震波传播过程中普遍存在的现象. 本文利用声波方程高阶有限差 分数值模拟技术精细刻画出波在均匀球状异常体中的传播过程.除再现了波前弥合现象 外，本文同时在前人工作基础上总结了波在异常体中的传播特征，分析了波前弥合现象与 地震波主频、异常体尺度之间的关系. 通过对绕射波、透射波波前能量的定量对比，总结 了波前弥合现象对地震层析成像的影响规律. 文中指出, 在强散射条件下, 射线层析方法 只能反演出高速异常体，有限频层析方法只能反演出大尺度异常体，低速小尺度异常体无 法用基于射线理论或有限频理论的透射走时层析成像方法反演出来; 最后通过地震层析 成像理论模型实验对以上规律的正确性进行了验证。本文同时根据波前弥合对 Banana-doughnut 现象进行了解释, 并对地震波的散射给出了新的理解.
\end{abstract}

关键词

有限频

菲涅尔体层析成像

散射条件

地震反演

波前弥合
基于射线理论的初至波走时层析成像利用观测 到的初至波到达时反演介质的属性信息. 目前该方 法在医学、天然地震学、地球动力学、油气勘探、工 程探测等众多领域中得到了成功的应用 ${ }^{[1 \sim 7]}$. 如在地 球动力学领域, 全球不同区域的地下速度结构主要 是通过地震波走时层析成像建立起来的, 这些认识 对于研究地球起源、地球内部物质交换过程以及板块 构造运动机制均有重要意义 ${ }^{[2,5,8,9]}$. 在勘探地震学领 域, 利用初至波或折射波走时反演近地表速度结 构 $^{[10 ~ 14]}$ 、利用反射波走时反演中深层速度 ${ }^{[6]}$ 、利用井 间透射波走时反演两井之间储层的精细结构 ${ }^{[4]}$ 、利用 VSP 资料中的下行或上行地震波走时反演井旁有限 区域的速度结构 ${ }^{[15]}$ 、利用叠前深度偏移进行剩余曲率 速度分析 ${ }^{[16,17]}$ 等.
然而随着研究的深入，基于射线理论的初至波 走时层析成像方法难以再满足对研究对象进行精细 反演的要求. 因此，近十几年来国内外学者对基于有 限频理论的菲涅尔体层析成像方法进行了深入的研 究 ${ }^{[12,18 ~ 22]}$. 走时菲涅尔体层析成像方法利用观测到 的初至波主能量到达时反演介质的属性信息，核函 数的求取是该方法的核心 ${ }^{[12]}$. $\mathrm{Wu}$ 和 Toksöz ${ }^{[23]}$, Woodward $^{[18]}$, Snieder 和 Lomax ${ }^{[19]}$, Marquering 等 ${ }^{[20]}$ 在理论上建立了 Rytov 波场与 Born 散射场之间的关 系, 并导出了相位、振幅扰动与 Born 散射场之间的 定量表达式. Marquering 等 ${ }^{[21]}$, Spetzler 和 Snieder ${ }^{[22,24]}$ 进一步分别从不同的角度出发建立了均匀介质情况 下单频、带限菲涅尔体走时层析核函数, 并描述了该 层析核函数的特点. 在这些工作的基础上 Dahlen 
等 ${ }^{[25,26]}$, Hung 等 ${ }^{[27]}$, Zhang 等 ${ }^{[28]}$ 沿用 Marquering 等 ${ }^{[20,21]}$ 的方法导出了非均匀介质情况下单频、带限菲 涅尔体走时层析核函数的计算方法, 并研究了该核 函数的特点. 研究发现, 三维走时菲涅尔体层析核函 数在中心射线路径上处处为零, 远离射线后又逐渐 增大, 然后又减小. Marquering 等 ${ }^{[21]}$ 首次将三维走时 菲涅尔体层析核函数中间为 “空” 的这种现象称为 Banana-doughnut 现象. 波前弥合 ${ }^{[29]}$ 是地震波传播过 程中普遍存在的现象. Hung 等 ${ }^{[27]}$ 利用波前弥合现象 对 Banana-doughnut 现象给出了很好的解释. Thore 和 Juliard $^{[30]}$, Nolet 和 Dahlen ${ }^{[29]}$ 同时指出波前弥合会对 观测数据造成很大影响, 尤其在菲涅尔体地震层析 成像中, 波前弥合会影响透射波走时的准确拾取.

本文在强散射条件下利用声波方程高阶有限差 分数值模拟方法再现了这种波前弥合现象, 并在前 人工作基础上分析了波在异常体中的传播特征, 分 析了波前弥合现象与地震波主频、异常体尺度之间的 关系. 通过对绕射波、透射波波前能量的定量对比， 总结了波前弥合现象对地震层析成像的影响规律, 并通过地震层析成像理论模型实验对这些规律进行 了验证. 本文最后指出, 只有利用包括走时在内的更 多信息才有可能得到介质的更高波数成分.

\section{1 层析方法介绍}

本文重点在于研究波前弥合现象对地震层析成 像的影响规律, 尤其是对射线层析成像与菲涅尔体 地震层析成像的影响, 因此首先对射线层析与菲涅 尔体层析成像方法进行简单介绍.

基于射线理论的初至波走时延迟 $\Delta \tau$ 可以表达 为方程(1)形式:

$$
\Delta \tau=\int_{\Gamma} \Delta s(r) \mathrm{d} r,
$$

其中, $\Delta s(r)$ 为射线路径 $\Gamma$ 上 $r$ 处的慢度扰动. 对方程(1) 进行离散即得到层析成像线性方程组. (1)式表明只 有射线路径上的点对接收的走时信息具有影响，而 且射线路径上的任意点对接收的走时信息具有相同 的影响权重 1 .

然而，根据地震波传播的有限频理论 ${ }^{[18 \sim 28]}$, 对 于某个特定震相的观测信息, 不仅射线路径上的点 对该信息具有影响, 中心射线领域上的其他点对接 收信息也具有影响, 而且空间不同位置的点对接收
信息的影响程度是不同的. 这种影响可以用核函数 $K(r)$ 表达为

$$
\Delta \sigma=\int_{V} K(r) \Delta s(r) \mathrm{d} r,
$$

上式中, $K(r)$ 反映了观测信息对介质扰动的敏感程度. 根据层析成像中所使用的观测信息的不同, $K(r)$ 可以 代表不同的核函数. 如 $\Delta \sigma$ 分别代表振幅扰动 $\Delta A$ 、走 时扰动 $\Delta \tau$ 与波形扰动 $\Delta u$ 时, 则 $K(r)$ 分别代表振幅层 析核函数 $K_{A}(r)$ 、走时层析核函数 $K_{T}(r)$ 与波形层析核 函数 $K_{U}(r) . V$ 为中心射线附近对初至信息贡献最大的 邻域范围, 通常选为第一菲涅尔体. 因此该方法也称 为菲涅尔体地震层析成像方法. 公式(2)中, 核函数 的计算与菲涅尔体边界的确定是方法的核心.

例如, 有限频地震波传播的振幅扰动 $\Delta A$ 可以表 达为

$$
\Delta A=\int_{V} K_{A}(r) \Delta s(r) \mathrm{d} r,
$$

其中, $K_{A}(r)$ 为振幅层析核函数; 同样, 空间慢度扰动 对接收的地震波走时也具有影响, 该影响可以用走 时核函数 $K_{T}(r)$ 来表达. 因此, 有限频地震波传播的 走时延迟 $\Delta \tau$ 可以表达为类似于方程(3)的形式:

$$
\Delta \tau=\int_{V} K_{T}(r) \Delta s(r) \mathrm{d} r .
$$

单频核函数的格林函数表达如公式(5)所示.

$$
\begin{aligned}
& K_{A}(r, \omega)=\frac{2 \omega^{2} A_{0}}{v_{0}(r)} \cdot \operatorname{Re}\left[\frac{G_{0}(g, r) u_{0}(r, s)}{u_{0}(g, s)}\right], \\
& K_{T}(r, \omega)=\frac{2 \omega}{v_{0}(r)} \cdot \operatorname{Im}\left[\frac{G_{0}(g, r) u_{0}(r, s)}{u_{0}(g, s)}\right],
\end{aligned}
$$

上式中, $A_{0}$ 为背景波场的振幅, $\omega$ 为圆频率, $\operatorname{Im}$ 表示取 复数的虚部, $\operatorname{Re}$ 表示取复数的实部. $G_{0}(g, r)$ 为无扰动 速度场 $v_{0}$ 中 $r$ 点在 $g$ 处的格林函数, $u_{0}(r, s)$ 为无扰动 速度场中 $s$ 点在 $r$ 点的波场, $u_{0}\left(g_{s} s\right)$ 为无扰动速度场中 $s$ 点在 $g$ 点的波场.

关于核函数的计算、核函数的性质及菲涅尔体边 界的确定可以参考刘玉柱等 ${ }^{[13]}$ 的文章, 这里不再赘 述.

\section{2 实验方法}

为了分析波的散射特征及波前弥合对地震层析 成像的影响, 本文采用高精度有限差分声波方程数 值模拟方法, 在二维均匀背景场模型中心设置一圆 
形异常体(图 1), 进行了六组井间单炮精细数值模拟 实验. 六组实验的模型规模、观测系统均相同. 实验 模型大小为 $1200 \mathrm{~m} \times 1200 \mathrm{~m}$, 离散网格大小为 $1 \mathrm{~m} \times 1$ $\mathrm{m}$. 激发点坐标为 $(200,600)$, 垂直接收井位于水平位 置 $1000 \mathrm{~m}$ 处. 在井中从地表到底部均匀布设 1200 个 检波点. 实验中子波主频分别取 30 与 $60 \mathrm{~Hz}$. 背景速 度均为 $2000 \mathrm{~m} \mathrm{~s}^{-1}$, 异常体速度分别取 1000 与 3000 $\mathrm{m} \mathrm{s}^{-1}$, 异常体直径分别取 40, 100 与 $200 \mathrm{~m}$, 时间采样 为 $0.1 \mathrm{~ms}$. 六组实验的具体参数如下:

(1) 主频波长 $33 \mathrm{~m}$, 异常体直径 $200 \mathrm{~m}$, 异常体 速度 $1000 \mathrm{~m} \mathrm{~s}^{-1}$;

(2) 主频波长 $33 \mathrm{~m}$, 异常体直径 $200 \mathrm{~m}$, 异常体 速度 $3000 \mathrm{~m} \mathrm{~s}^{-1}$;

(3) 主频波长 $66 \mathrm{~m}$, 异常体直径 $100 \mathrm{~m}$, 异常体 速度 $1000 \mathrm{~m} \mathrm{~s}^{-1}$;

(4) 主频波长 $66 \mathrm{~m}$, 异常体直径 $100 \mathrm{~m}$, 异常体 速度 $3000 \mathrm{~m} \mathrm{~s}^{-1}$;

（5）主频波长 $66 \mathrm{~m}$, 异常体直径 $40 \mathrm{~m}$, 异常体速 度 $1000 \mathrm{~m} \mathrm{~s}^{-1}$;

(6) 主频波长 $66 \mathrm{~m}$, 异常体直径 $40 \mathrm{~m}$, 异常体速 度 $3000 \mathrm{~m} \mathrm{~s}^{-1}$.

六组实验的理论模型如图 1 所示.

\section{3 实验结果与分析}

模拟过程中保存全波场信息，以方便对绕射波、 透射波的波前振幅进行定量对比，并记录地震波在 异常体附近的传播过程(图 2); 同时保存井间观测记 录(如图 3)以提取初至信息, 以分析波前弥合现象对 地震层析成像的影响.

以上实验不仅证实了波前弥合现象的存在，而 且通过观察地震波在异常体附近的传播过程，我们 可以得到以下一些认识(其中部分结论是对前人认识 结果的进一步印证):

(1) 波前弥合是地震波传播过程中普遍存在的 现象, 该现象的表现与地震波的频带范围、异常体的 空间尺度、异常值的大小、异常体的位置等因素有 关 ${ }^{[27]}$. 相对于地震波波长, 异常体尺寸越大波前断裂 越强, 弥合越慢, 异常体尺寸越小, 波前断裂越弱, 弥合越快. 相对于背景场速度, 异常体异常值越大波 前断裂越强，弥合越慢，反之亦然.

（2）透射波在正负异常体中的传播现象不同，低 速异常体对透射波有聚焦效应，高速异常体对透射 波有散焦效应。该聚焦与散焦效应进而会导致波穿 过正负异常体后弥合现象的不对称性(图 2) ${ }^{[27,29]}$.
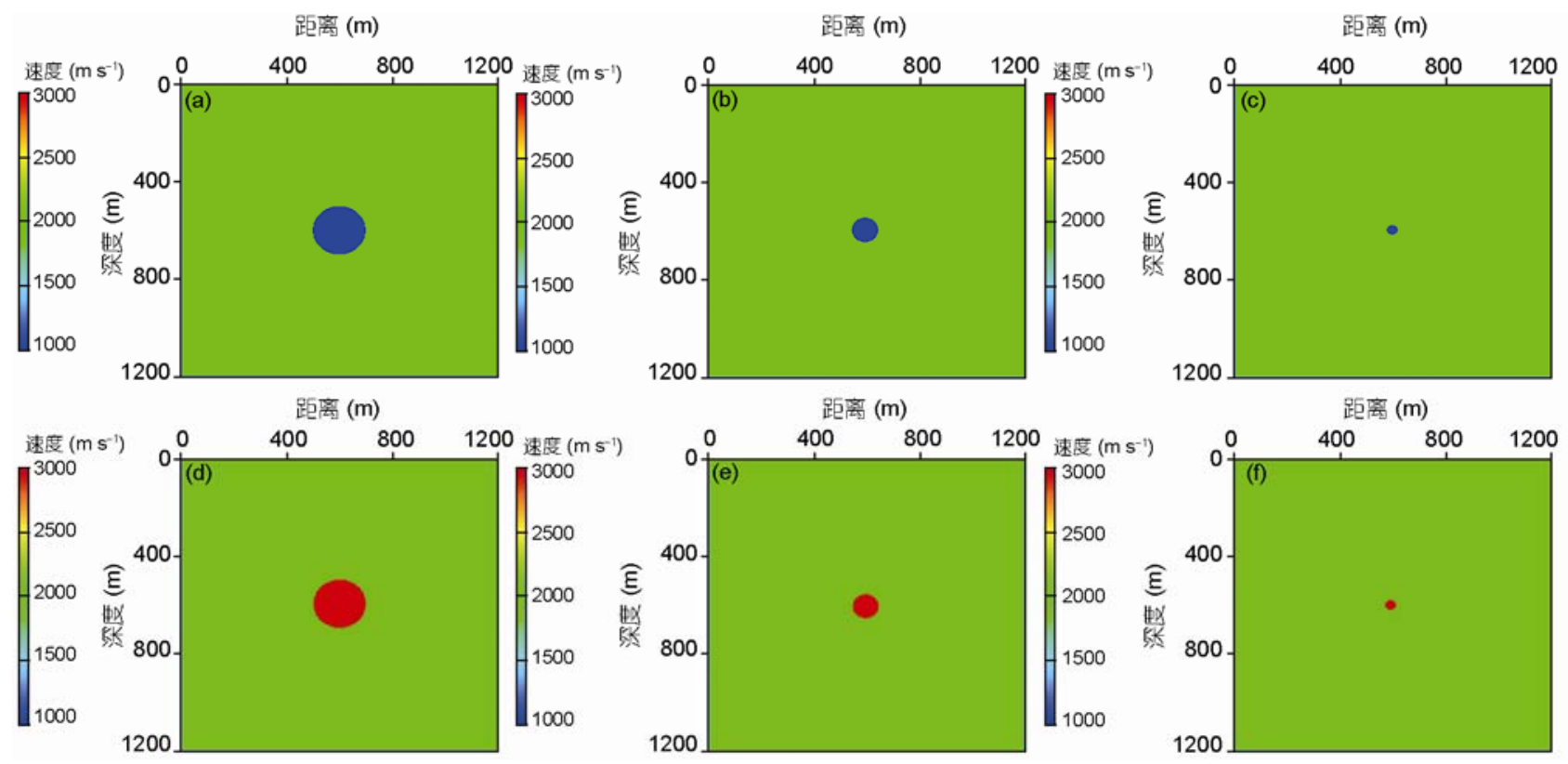

图 1 六组实验的理论模型

低速异常体((a) (c))速度为 $1000 \mathrm{~m} \mathrm{~s}^{-1}$, 高速异常体 $((\mathrm{d}) \sim(\mathrm{f}))$ 速度为 $3000 \mathrm{~m} \mathrm{~s}^{-1}$, 均匀背景速度为 $2000 \mathrm{~m} \mathrm{~s}^{-1}$; 异常体直径从左至右分别为 200 , 100 与 $40 \mathrm{~m}$ 

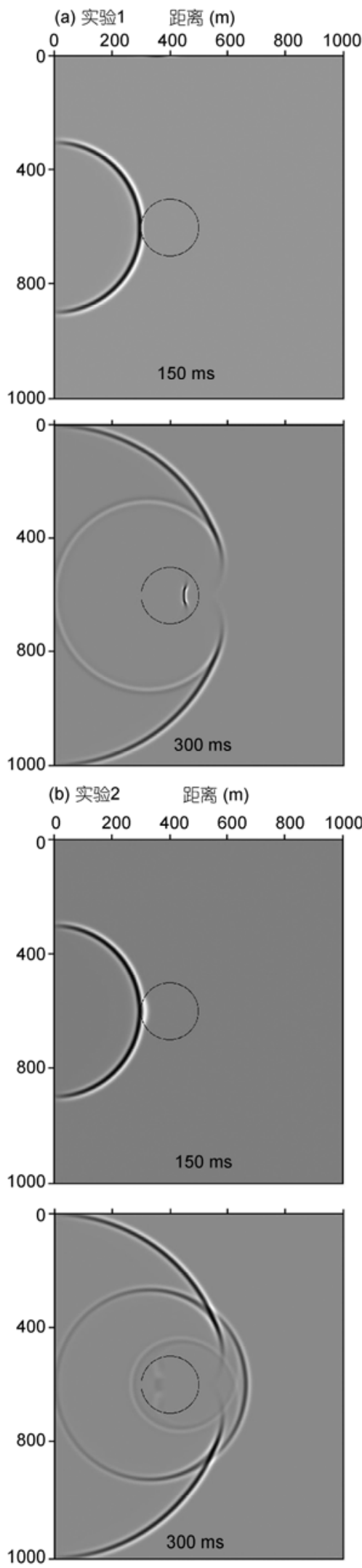
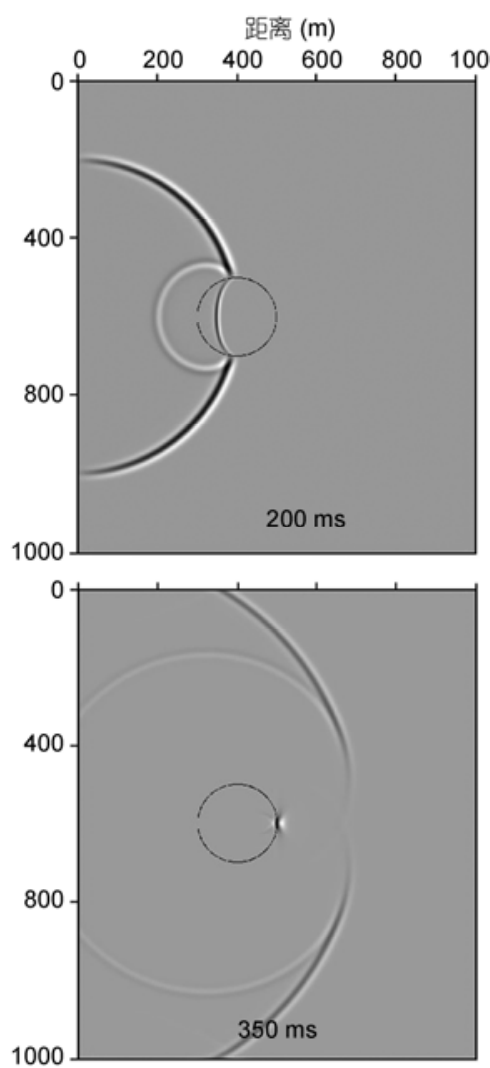

距离 $(m)$
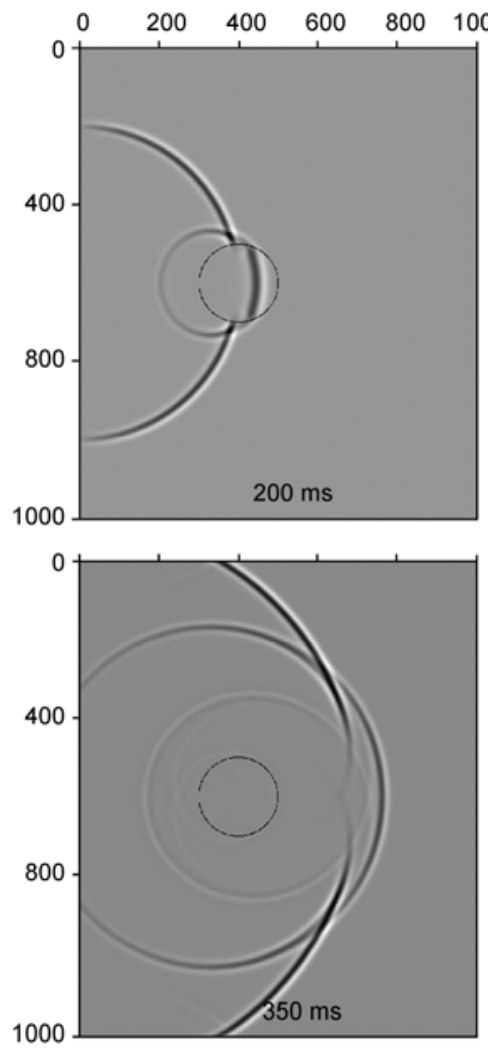
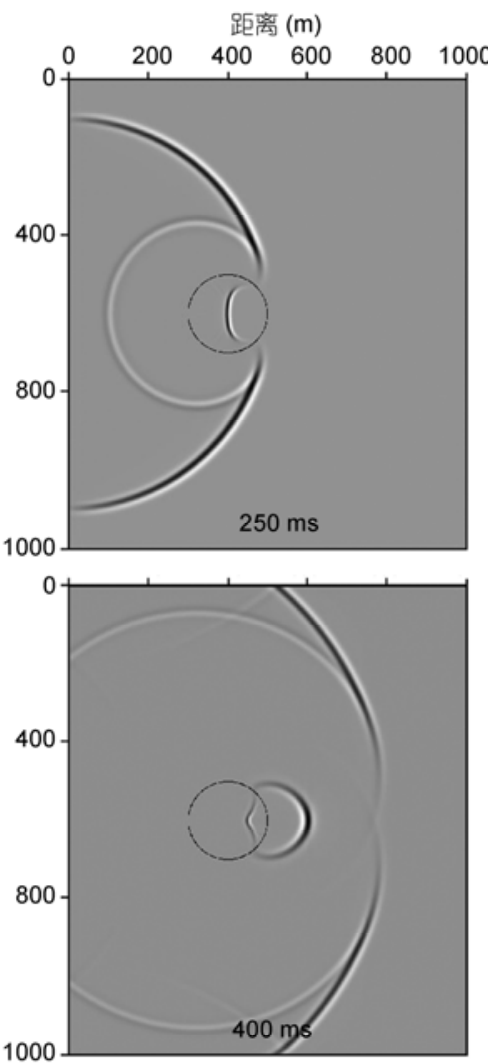

1000

距离 $(m)$
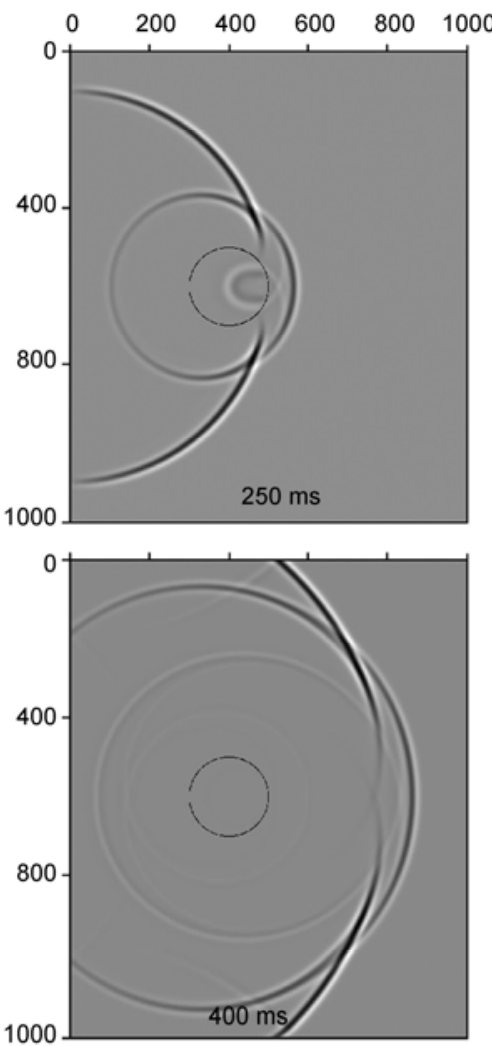

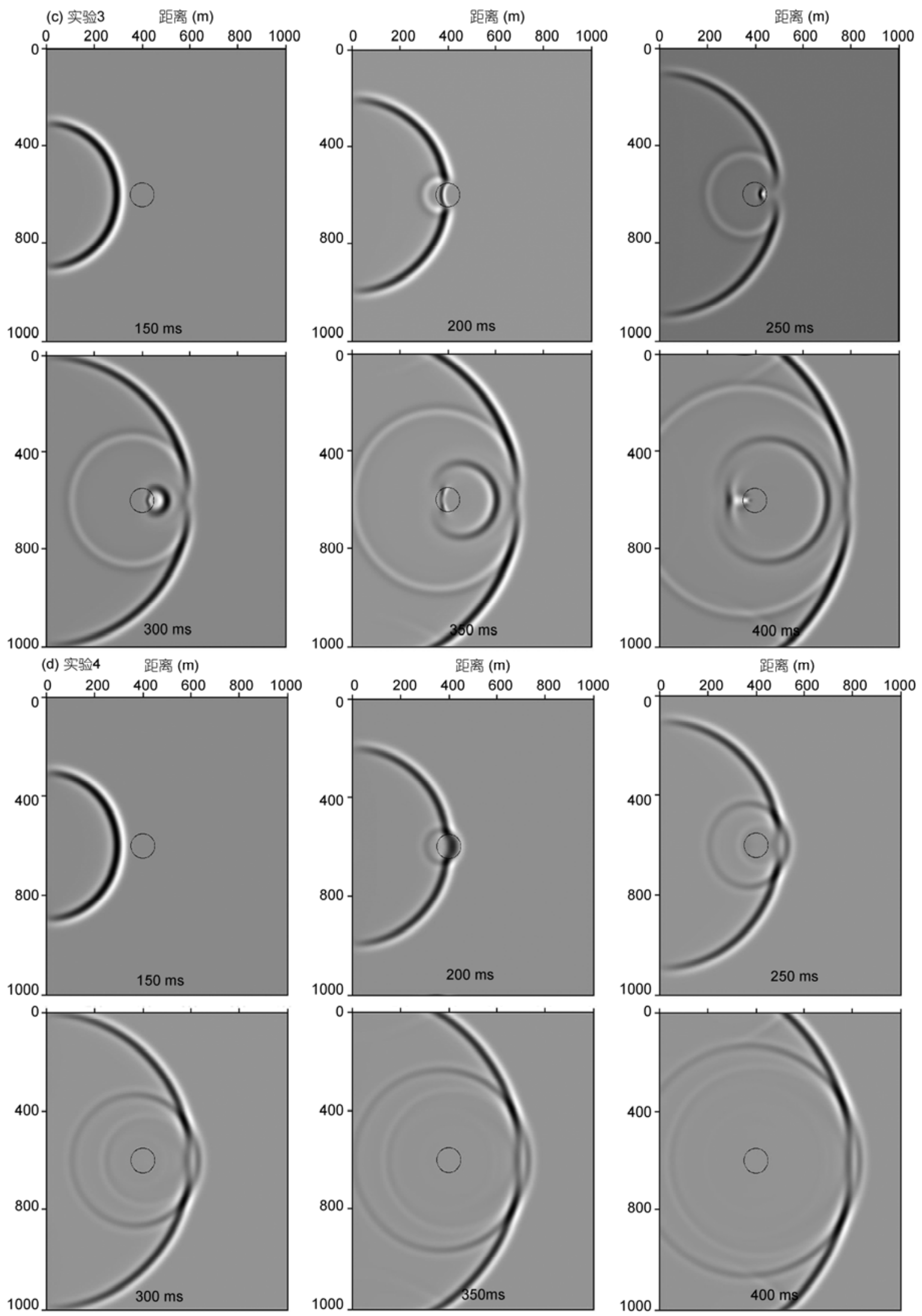

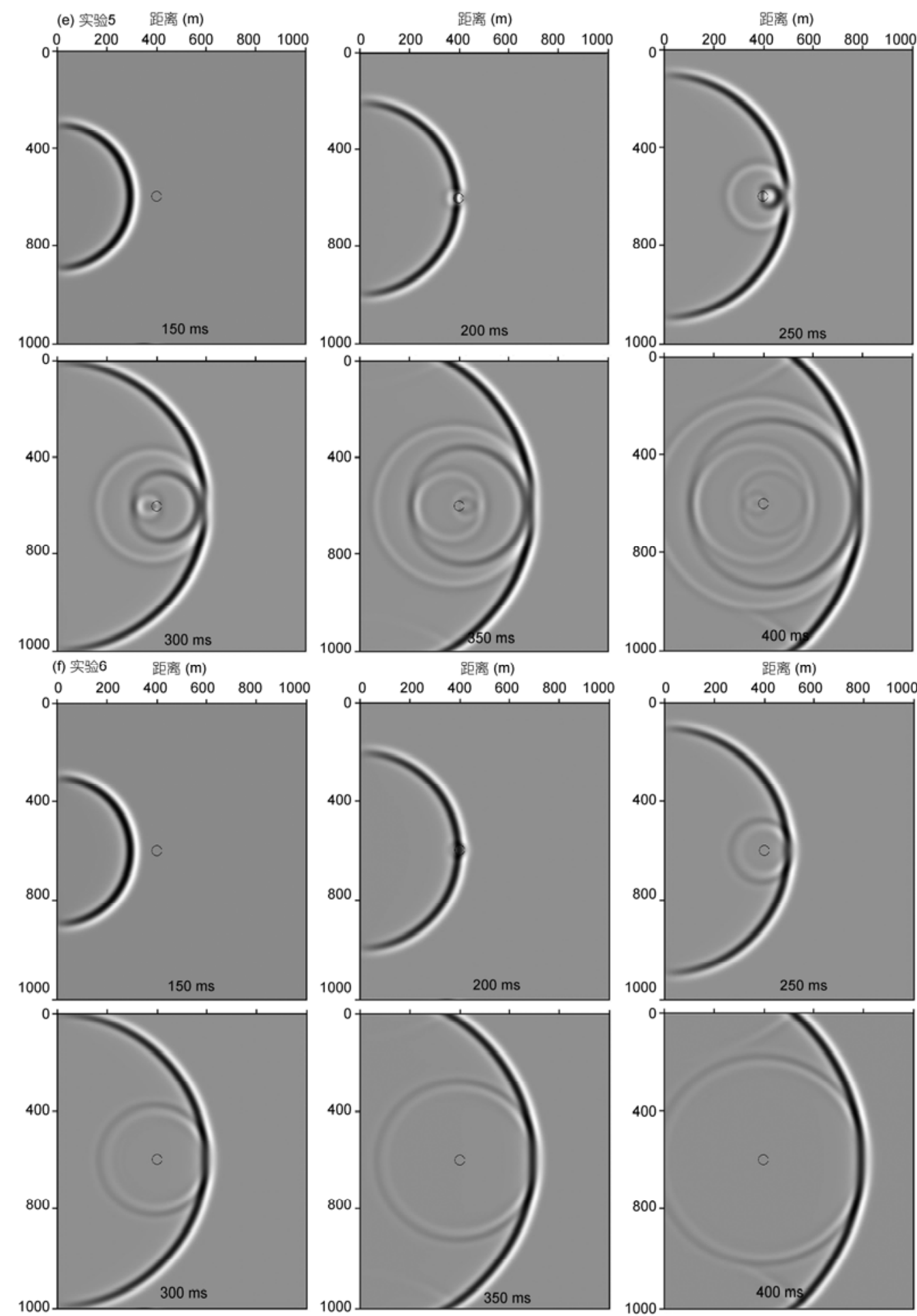

图 2 波场快照

(a) 实验 1(主频波长 $33 \mathrm{~m}$, 异常体直径 $200 \mathrm{~m}$, 异常体速度 $1000 \mathrm{~m} \mathrm{~s}^{-1}$ ) 波场快照; (b) 实验 2(主频波长 $33 \mathrm{~m}$, 异常体直径 $200 \mathrm{~m}$, 异常体速度 $3000 \mathrm{~m} \mathrm{~s}^{-1}$ )波场快照; (c) 实验 3(主频波长 $66 \mathrm{~m}$, 异常体直径 $100 \mathrm{~m}$, 异常体速度 $1000 \mathrm{~m} \mathrm{~s}^{-1}$ )波场快照; (d) 实验 4(主频波长 $66 \mathrm{~m}$, 异常体直径 $100 \mathrm{~m}$, 异常体速度 $3000 \mathrm{~m} \mathrm{~s}^{-1}$ ) 波场快照; (e) 实验 5(主频波长 $66 \mathrm{~m}$, 异常体直径 $40 \mathrm{~m}$, 异常体速度 $1000 \mathrm{~m} \mathrm{~s}^{-1}$ ) 波场快照; (f) 实验 6(主频波长 $66 \mathrm{~m}$, 异常体直径 $40 \mathrm{~m}$, 异常体速度 $3000 \mathrm{~m} \mathrm{~s}^{-1}$ )波场快照 

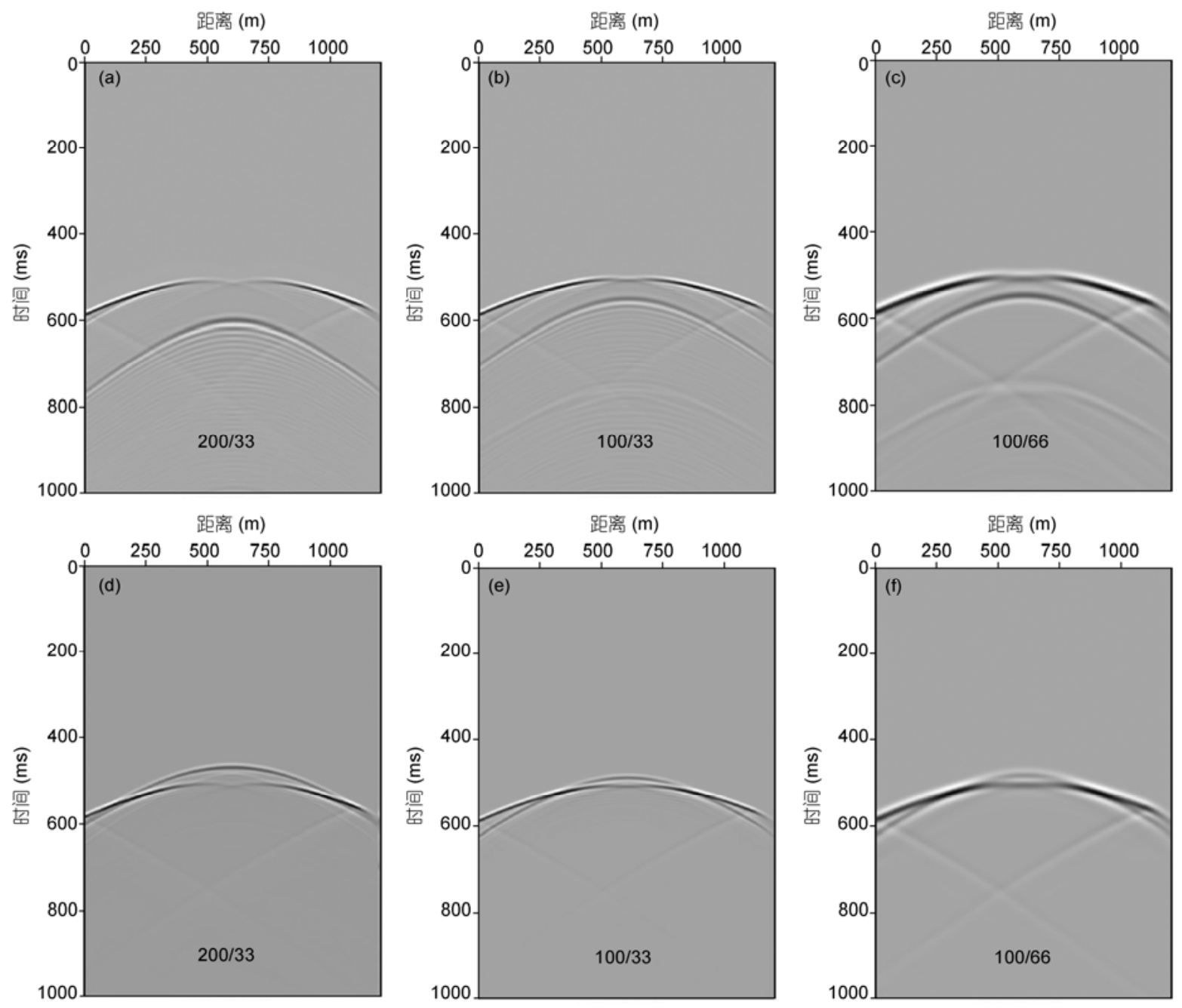

图 3 低速异常((a) (c)) 与高速异常((d) (f)) 实验下不同异常体尺度/地震波主频波长比对应的井间观测记录

（3）地震波在异常体中会反复震荡, 震荡过程中 不断散射能量, 在传播方向与反传播方向上表现为两 个次级源激发; 当异常体很小时, 前后激发的波前面 重合, 异常体表现为一个单一的次级源(图 2(e), (f)).

（4）异常体尺寸越小绕射现象越明显，绕射波的 能量越强, 透射波的能量越弱. 反之, 异常体尺寸越 大, 透射现象越明显, 透射波能量越强, 绕射波的能 量越弱. 这一点可以解释三维菲涅尔体层析核函数 的 Banana-doughnut 现象. 因为异常体尺寸很小时, 绕射波的弥合波前面能量很强，导致“拾取”到的初 至走时是弥合走时而非透射波走时，而弥合走时基 本没有延迟, 因此中心射线上的异常体对初至走时 影响为零.

(5) 根据地震层析成像的基本原理(即只有经过
异常体的地震波才携带了异常体的信息)还可以给出 下述结论: 传统的基于射线理论的初至波层析成像 方法可以反演出高速异常体, 因为高速异常体的透 射走时总是可以被拾取到; 而基于有限频理论的菲 涅尔体层析成像方法可以反演出大尺度异常体，因 为大尺度异常体的透射波能量较强, 导致其容易被 “拾取”到；对于小尺度的低速异常体，基于射线理论 与有限频理论的地震走时层析成像方法无法将其反 演出来，因为其透射波在弥合的绕射波之后，且其能 量较弱，导致其不容易被“拾取”到; 虽然波前弥合影 响了透射波相位的准确拾取，但无论是透射波还是 弥合的绕射波，其波前面的动力学特征都发生了变 化，因此，只要利用包括走时在内的更多信息如振幅 与波形, 或将初至波与后续波同时利用起来就有可 
能得到所有异常体的信息.

需要注意的是, 本文散射实验中正负异常体的 异常值为 50\%, 大于 Jocker 等 ${ }^{[31]}$ 给出的 $15 \%$ 弱散射 条件. 同时, 本文实验中散射因子 $3.8<k a<38$. 根据 Nolet $^{[32]}$ 对散射区域的划分, 该散射介于较强的 Mie 散射与前向散射之间, 大部分属于前向散射. 因此,
本文给出的最后一个散射实验结论只在强散射条件 下有效。在弱散射条件下，透射波波前面会对绕射波 的弥合波前面产生微小影响, 该影响正是有限频理 论 ${ }^{[27,29]}$ 所能够预测的, 因此有限频理论对弱散射条 件下的小的异常体仍然敏感, 这也正是菲涅尔体层 析优于传统射线层析之处.

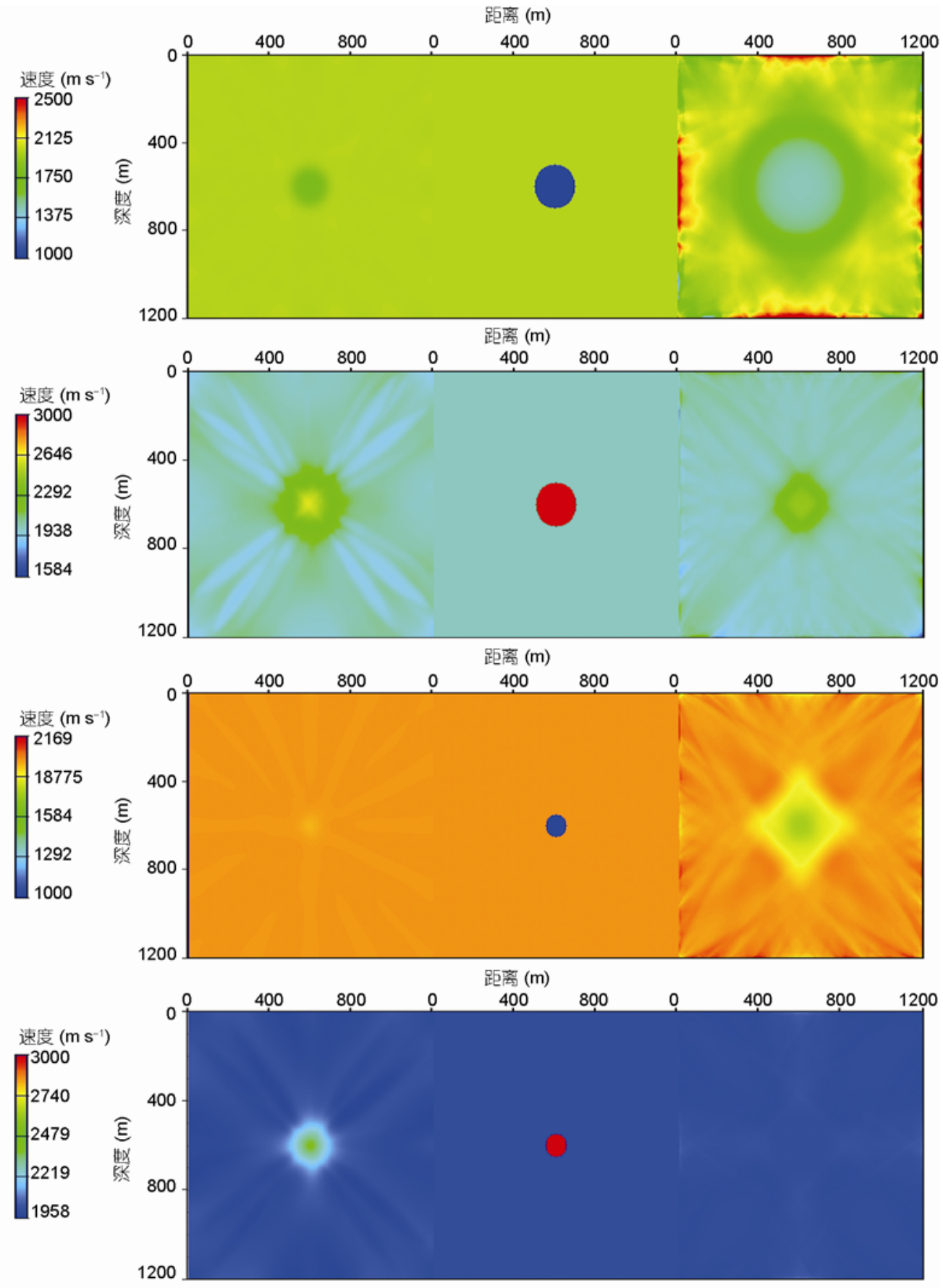

图 4 四组实验的理论模型(中)及其对应的射线走时层析反演结果(左)与菲涅尔体走时层析反演结果(右) 


\section{4 理论模型验证}

为了进一步验证波前弥合现象对层析成像的上 述影响, 本文做了如下四组单异常体的射线层析与 菲涅尔体层析成像对比实验. 四组实验中的理论模 型与图 1 相同，具体实验参数如下:

（1）异常体直径 $200 \mathrm{~m}$, 异常体速度 $1000 \mathrm{~m} \mathrm{~s}^{-1}$;

（2）异常体直径 $200 \mathrm{~m}$ ，异常体速度 $3000 \mathrm{~m} \mathrm{~s}^{-1}$;

（3）异常体直径 $100 \mathrm{~m}$, 异常体速度 $1000 \mathrm{~m} \mathrm{~s}^{-1}$;

（4）异常体直径 $100 \mathrm{~m}$, 异常体速度 $3000 \mathrm{~m} \mathrm{~s}^{-1}$. 实验中采用声波方程高精度有限差分数值模拟 方法计算地震波场. 为了提高反演精度, 采用底部激 发地表接收与左边激发右边接收的交叉观测系统. 垂直与水平方向上均为等间隔的 241 炮激发、241 道 接收, 炮距、道距均为 $5 \mathrm{~m}$. 在模拟记录上拾取初至 走时进行射线走时层析成像. 将模拟记录作为观测 数据, 采用互相关方法计算初至波主能量走时残
差 $^{[20,21]}$, 进而进行菲涅尔体走时层析成像反演. 两种 方法均采用 $2000 \mathrm{~m} \mathrm{~s}^{-1}$ 的匀速初始模型. 四组实验反 演结果如图 4 所示.

由上述实验结果不难看出，对于射线初至波走 时层析反演方法, 高速大尺度异常体与高速小尺度 异常体能被较好地反演了出来, 低速大尺度异常体 反演的不准确，低速小尺度异常体由于受严重的波 前弥合现象影响, 基本反演不出来. 我们还可以发现, 虽然高速异常体可以被较好地反演出来，但异常体 尺度反演的较差, 这是由高速异常体的散焦效应导 致的(图 5(b)); 低速异常体虽然反演精度低, 但可以 得到较好的异常体尺度, 这是由低速异常体的聚焦 效应导致的(图 5(a)); 低速异常体实验中反演得到的 低速异常值来自于波前弥合走时延迟的贡献(图 6(a)), 异常体越大波前弥合走时延迟越大，当低速异常体尺 寸很大时, 波前弥合趋于消失, 我们便可以拾取得到 透射波的初至走时, 进而将低速异常体也反演出来.

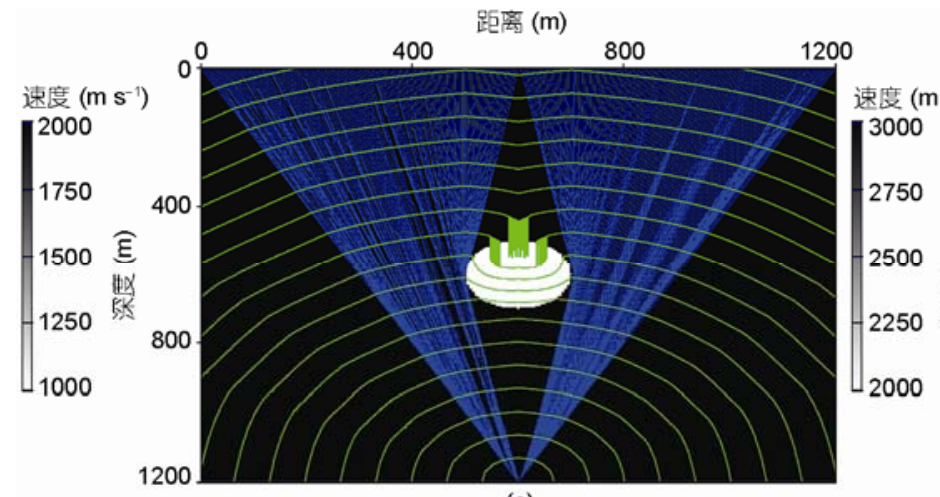

(a)

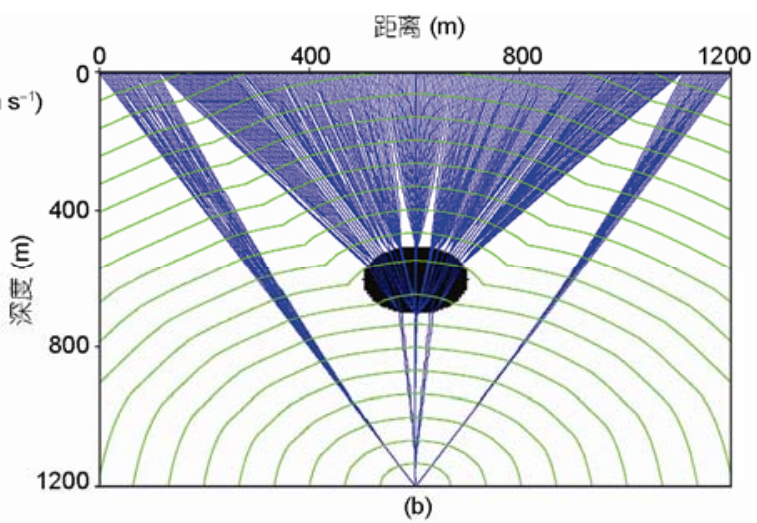

(b)

图 5 低速异常体(a)与高速异常体(b)的射线路径图(蓝线) 绿线为波震面
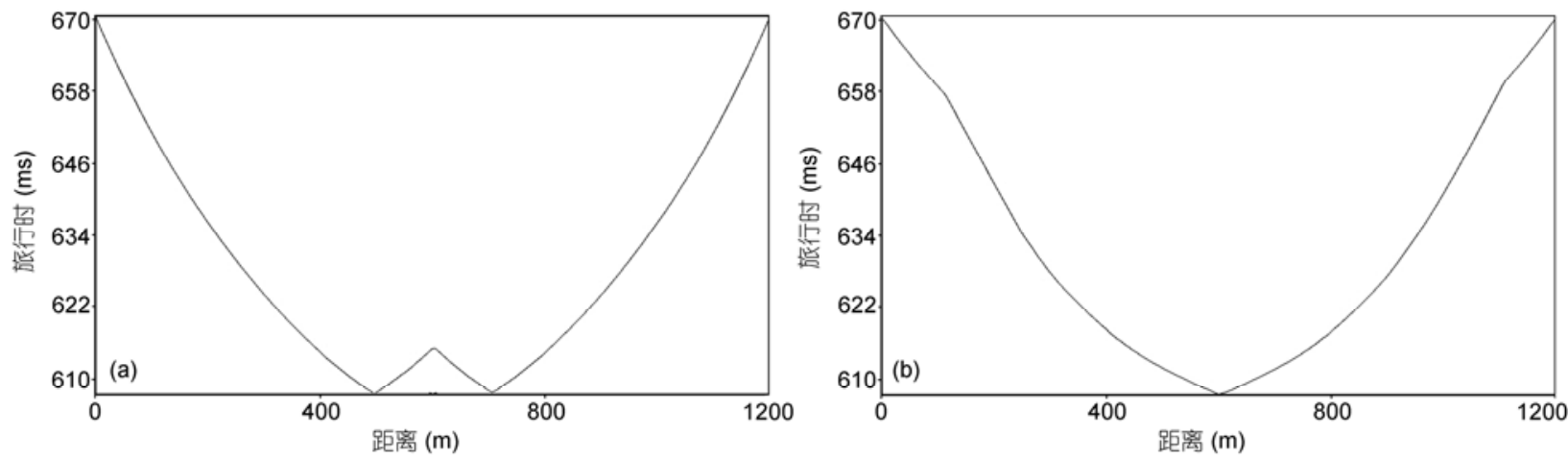

图 6 图 5 中低速异常体(a)与高速异常体(b)地表接收的初至走时图 
对于菲涅尔体地震走时层析成像方法, 高速大 尺度异常体与低速大尺度异常体被较好地反演了出 来, 低速小尺度异常体反演的不够准确, 高速小尺度 异常体基本没有被反演出来. 反演出来的部分低速 异常值同样来自于波前弥合走时延迟的贡献.

上述实验结果印证了本文总结的波前弥合对地 震层析成像的影响规律.

\section{5 结论与讨论}

与 Banana-doughnut 现象一样, 波前弥合属于一 种波动现象. 正是由于波动具有这种属性, 我们才能
够从观测到的波场中得到介质的背景场信息. 通过 本文的正演模拟与层析反演实验，我们也可以认识 到波前弥合对层析反演有严重的负面影响. 最直接 的负面影响是绕射波与透射波的相互干涉影响了透 射波的准确拾取, 导致最终反演, 尤其是射线层析的 反演结果不对或精度较低. 在这种情况下, 为了提高 反演精度，除利用振幅、波形等更多信息或利用后续 波至采用波形反演方法外, 还可以通过提高初始模 型的精度以降低速度异常值或散射因子的大小, 使 得强散射条件变为弱散射条件, 那么基于有限频理 论的菲涅尔体地震层析成像方法的优点就可以得到 发挥.

\section{参考文献}

1 Sheriff R E, Geldart L P. Exploration Seismology. Cambridge: Cambridge University Press, 1982

2 Dziewonski A. Mapping the lower mantle: Determination of lateral heterogeneous in P-velocity up to degree and order 6. J Geophys Res, 1984, 89: 5929-5952

3 Nolet G. Seismic wave propagation and seismic tomography. In: Nolet G, ed. Seismic Tomography with Applications in Global Seismology and Exploration Geophysics. Dordrecht: D. Reidel Publishing Corporation, 1987

4 Harris J M, Nolen-Hoeksema R C, Langan R T, et al. High-resolution crosswell imaging of a west Texas carbonate reservoir: Part 1-Project summary and interpretation. Geophysics, 1995, 60: 667-681

5 Pulliam R J, Vasco D W, Johnson L R. Tomographic inversions for mantle P-wave velocity structure based on the minimization and norms of International Seismological Centre traveltime residuals. J Geophys Res, 1993, 98: 699-734

6 Billette F, Lambaré G. Velocity macro-model estimation from seismic reflection data by stereo-tomography. Geophys J Int, 1998, 135: 671-680

7 Aki K, Richards P G. Quantitative Seismology. 2nd ed. Herndon: University Science Books, 2002

8 洪学海, 朱介寿, 曹家敏, 等. 中国大陆地壳上地幔 S 波品质因子三维层析成像. 地球物理学报, 2003, 46: 642-651

9 刘福田, 吴华. 中国大陆及其邻近地区的地震层析成象. 地球物理学报, 1989, 32: 281-291

10 Zhu X H, Sixta D P, Angstman B G. Tomostatics: Turning-ray tomography+static corrections. Leading Edge, 1992, 11: 15-23

11 李录明, 罗省贤, 赵波. 初至波表层模型层析反演. 石油地球物理勘探, 2000, 35: 559-564

12 Liu Y Z, Dong L G, Wang Y W, et al. Sensitivity kernels for seismic Fresnel volume tomography. Geophysics, 2009, 74: U35-U46

13 刘玉柱, 董良国, 王毓玮, 等. 初至波菲涅尔体地震层析成像. 地球物理学报, 2009, 52: 2310-2320

14 刘玉柱, 董良国. 初至波走时层析中的正则化方法. 石油地球物理勘探, 2007, 42: 682-685, 698

15 Chen X H, Mu Y G. Nonlinear wave equation inversion of VSP data. The First International Conference "Inverse Problems: Modeling and Simulation", 2002 July 14-21, Fethiye, Turkey

16 Al-Yahya K. Velocity analysis by iterative profile migration. Geophysics, 1989, 54: 718-729

17 Liu Z Y, Bleistein N. Migration velocity analysis: Theory and an iterative algorithm. Geophysics, 1995, 60: 142-153

18 Woodward M J. Wave-equation tomography. Geophysics, 1992, 57: 15-26

19 Snieder R, Lomax A. Wavefield smoothing and the effect of rough velocity perturbations on arrival times and amplitudes. Geophys J Int, 1996, 125: 796-812

20 Marquering H, Nolet G, Dahlen F A. Three-dimensional waveform sensitivity kernels. Geophys J Int, 1998, 132: 521-534

21 Marquering H, Dahlen F A, Nolet G. Three-dimensional sensitivity kernels for finite-frequency traveltimes: The banana-doughnut paradox. Geophys J Int, 1999, 137: 805-815

22 Spetzler J, Snieder R. The Fresnel volume and transmitted waves. Geophysics, 2004, 69: 653-663 
23 Wu R S, Toksöz M N. Diffraction tomography and multisource holography applied to seismic imaging. Geophysics, 1987, 52: 11-25

24 Spetzler J, Snieder R. The effect of small-scale heterogeneity on the arrival time of waves. Geophys J Int, 2001, 145: 786-796

25 Dahlen F A, Hung S H, Nolet G. Fréchet kernels for finite-frequency traveltimes-I. Theory. Geophys J Int, 2000, 141: 157-174

26 Dahlen F A. Finite-frequency sensitivity kernels for boundary topography perturbations. Geophys J Int, 2005, 162: 525-540

27 Hung S H, Dahlen F A, Nolet G. Wavefront healing: A banana-doughnut perspective. Geophys J Int, 2001, 146: 289-312

28 Zhang Z G, Shen Y, Zhao L. Finite-frequency sensitivity kernels for head waves. Geophys J Int, 2007, 171: 847-856

29 Nolet G, Dahlen F A. Wave front healing and the evolution of seismic delay times. J Geophys Res, 2000, 105: 19043-19054

30 Thore P D, Juliard C. Fresnel zone effect on seismic velocity resolution. Geophysics, 1999, 64: 593-603

31 Jocker J, Spetzler J, Smeulders D, et al. Validation of first-order diffraction theory for the traveltimes and amplitudes of propagating waves. Geophysics, 2006, 71: 167-177

32 Nolet G. A Breviary of Seismic Tomography, Chapter 4. Cambridge: Cambridge University Press, 2008 